\title{
Smoking cessation: an economic analysis and review of varenicline
}

This article was published in the following Dove Press journal:

ClinicoEconomics and Outcomes Research

19 June 2009

Number of times this article has been viewed

\author{
Michele A Faulkner \\ Creighton University School \\ of Pharmacy and Health Professions, \\ Omaha, NE, USA
}

\begin{abstract}
Despite efforts to decrease tobacco use, smoking continues to be a leading cause of preventable morbidity and premature death. The associated economic burden is substantial, both in the form of direct costs (healthcare expenditures) and indirect costs (lost productivity), regardless of whether the burden is assessed from the standpoint of an employer, a health plan, or society as a whole. Cessation programs are considered among the most cost-effective in healthcare, and are often used as a benchmark for other medical interventions. This analysis specifically considers the cost-effectiveness of varenicline, a novel $\alpha_{4} \beta_{2}$ partial agonist used for smoking cessation, in comparison to other approved therapies. Clinical trial data have demonstrated that varenicline has the ability to decrease cravings and withdrawal symptoms, and lessen positive reinforcement associated with smoking. Varenicline's novel mechanism has translated into superior efficacy in comparison to other available therapies. For this reason, despite an initial cost that typically exceeds that of other medications, varenicline is a costeffective option for smoking cessation.
\end{abstract}

Keywords: cost-effectiveness, economic analysis, smoking cessation, varenicline

According to the World Health Organization, more than one billion persons worldwide routinely smoke, and despite efforts to aid people in their attempts to quit and the provision of education about the dangers of tobacco use, it is estimated that the number of people who smoke will climb to more than 1.6 billion by the year 2025. ${ }^{1}$ The economic burden associated with smoking affects all of society, and though the perspective from which the burden is analyzed alters the impact, the costs remain substantial. Direct medical costs, which include such things as medications, medical services (both inpatient and outpatient), institutional care, diagnostics, and ER visits, were estimated to be greater than US $\$ 75.5$ billion in 1998, while indirect costs, such as lost wages and decreased job performance, contributed another US $\$ 92$ billion. ${ }^{2}$ Individuals who smoke consume $40 \%$ more health care costs than nonsmokers. ${ }^{3}$ Tobacco use is the leading cause of preventable illness and premature death. ${ }^{4}$

\section{Treatment options for smoking cessation}

Because it is well documented that quit attempts are substantially more successful when pharmacotherapy is employed, experts advise that medications be used unless there is a specific contraindication for doing so. ${ }^{5}$ Medications approved by the US Food and Drug Administration (FDA) for use in the treatment of nicotine dependence include various 
nicotine replacement therapies (NRT) including patches, lozenges, inhalers, nasal spray and gum, the antidepressant bupropion, and varenicline (Chantix ${ }^{\circledR}$; Pfizer, New York, USA). Other medications sometimes used for smoking cessation therapy include clonidine and nortriptyline, though neither is FDA approved for this purpose.

Nicotine replacement works by acting on the same nicotinic receptors in the central nervous system (CNS) that are stimulated when cigarette smoke is inhaled. Nicotine binding to these receptors results in the release of dopamine and norepinephrine. Stimulation of dopamine receptors in particular is believed to be responsible for the properties of tobacco smoke that ultimately lead to addiction. ${ }^{6}$ The acetylcholine receptors on dopaminergic neurons in the mesolimbic system in the brain are known to play a role in pleasure and reinforcement. Because nicotine exerts its effects on the receptor for a prolonged time period, upregulation occurs. This leads to desensitization of the receptor, and results in physical dependence, tolerance, and ultimately to symptoms of withdrawal should the smoker quit without an aid to offset these effects. ${ }^{7}$ NRT is used primarily to decrease symptoms of withdrawal while the smoker is gradually weaned from the physical addiction. Unlike the inhalation of nicotine contained in smoke, which allows for nearly immediate effects on receptors and virtually instant satisfaction for the smoker, replacement therapies have a slower onset, allowing the user to become less accustomed to immediate reinforcement. ${ }^{8}$ By decreasing withdrawal symptoms, replacement therapy allows the smoker to focus on necessary changes in behavior. NRT has been shown in clinical trials to increase quit rates by up to twice that noted in smokers who attempt to quit without pharmacologic therapy. ${ }^{5}$ In general, trials have not demonstrated a substantial difference in efficacy among the various available dosage forms, and thus the decision about which one to use is up to the smoker and the prescriber. ${ }^{4}$

Bupropion is also used in an oral sustained-release (SR) formulation as an aid to smoking cessation. It was the first non-nicotine medication to be FDA approved for this purpose. Previously, the drug had been marketed as an antidepressant medication (Wellbutrin ${ }^{\circledR}$; GlaxoSmithKline, North Carolina, USA). Bupropion is now available in a generic formulation. The drug is a weak inhibitor of dopamine and norepinephrine uptake, and may have some ability to cause the release of dopamine and inhibit nicotinic receptors. ${ }^{4,9}$ Clinical trials have demonstrated the ability of bupropion to approximately double the successful quit rate compared to no therapy. ${ }^{5}$

In 2008, the US Department of Health and Human Services put forth revised clinical practice guidelines for treating the use of and dependence on tobacco. ${ }^{5}$ The guidelines place both nicotine replacement and bupropion in the category reserved for first-line agents based on their safety profiles and the amount of robust trial data available for both. Clonidine (despite having level A strength of evidence for efficacy and clinical trial data which suggest its use can double quit rates) was classified as a second-line agent due to lack of FDA approval, its side-effect profile, and the need for a specific dosing regimen for use in smoking cessation. Similarly, nortriptyline was classified as second line, again due to lack of FDA approval, its side-effect profile, and a limited number of clinical studies with small sample sizes (strength of evidence $=$ level B). ${ }^{5}$ Varenicline was not included, presumably because it was still a relatively new agent at the time the guidelines were being formulated.

A novel oral medication for the treatment of nicotine addiction, varenicline received FDA approval in May 2006. The drug is a derivative of cytisine, a substance found to be useful in controlling cravings for nicotine when the leaves of the golden rain tree (Koelreuteria paniculata) were substituted for tobacco during World War II. ${ }^{6}$ In fact, cytisine is used in Eastern Europe as an aid for smoking cessation. Structurally, varenicline is similar to nicotine. The drug is a partial agonist at the $\alpha_{4} \beta_{2}$ nicotinic receptor. Through its partial binding, it mimics the effects of nicotine, though the reinforcing effects of varenicline are less. Its nicotinic receptor binding also results in the release of dopamine and a decrease in cravings. By preventing receptor access to nicotine, varenicline is able to block the expected reinforcing effects. ${ }^{4}$ Typically, the drug is given at a dose of $0.5 \mathrm{mg}$ daily for the first 3 days, $0.5 \mathrm{mg}$ twice daily for the next 4 days, and then on the eighth day (generally the scheduled quite date), the drug is titrated up to its final dose of $1 \mathrm{mg}$ twice daily. Because the recommended schedule to set a quit date is 8 days after initiation of therapy, it has been hypothesized that positive reinforcement may actually decrease with each cigarette smoked during the lead-in week. ${ }^{10}$

\section{Efficacy of varenicline}

Study data have shown varenicline to be superior to placebo for smoking cessation. ${ }^{11,12}$ In addition, multiple trials have demonstrated varenicline to be efficacious when compared to other active treatments. Though an exhaustive review of efficacy trials is beyond the scope of this publication, available data from trials comparing varenicline to other medication approved for smoking cessation are summarized below (Table 1).

A phase II trial by Nides et al was designed to compare three different doses of varenicline to bupropion and placebo. ${ }^{13}$ Six hundred thirty-eight subjects were enrolled and 
Table I Fifty-two week continuous abstinence rates with varenicline versus bupropion SR or nicotine replacement

\begin{tabular}{|c|c|c|c|c|}
\hline Study & Design & Treatment groups & Abstinence at 52 weeks & $P$ value \\
\hline \multirow[t]{2}{*}{ Nides et $\mathrm{al}^{13}$} & $\mathrm{R}, \mathrm{DB}, \mathrm{PC}$ & varenicline I $\mathrm{mg}$ bid (7 weeks) & $14.4 \%$ & $<0.01$ \\
\hline & $n=638$ & bupropion SR I 50 mg bid (7 weeks) & $6.3 \%$ & \\
\hline \multirow[t]{2}{*}{ Gonzales et al ${ }^{15}$} & $R, D B, P C$ & varenicline I mg bid ( 12 weeks) & $21.9 \%$ & 0.057 \\
\hline & $\mathrm{n}=1025$ & bupropion SR I50 mg bid (12 weeks) & $16.1 \%$ & \\
\hline \multirow[t]{2}{*}{ Jorenby et $\mathrm{al}^{16}$} & $R, D B, P C$ & varenicline I mg bid ( 12 weeks) & $23 \%$ & $<0.001$ \\
\hline & $\mathrm{n}=1027$ & bupropion SR I50 mg bid (12 weeks) & $14.6 \%$ & \\
\hline \multirow[t]{2}{*}{ Aubin et $\mathrm{al}^{14}$} & $\mathrm{R}, \mathrm{OL}$ & varenicline I mg bid (12 weeks) & $26.1 \%$ & 0.056 \\
\hline & $\mathrm{n}=746$ & $\begin{array}{l}\text { TN } 21 \mathrm{mg} / \text { day }(6 \text { weeks), } 14 \mathrm{mg} / \text { day } \\
(2 \text { weeks), } 7 \mathrm{mg} / \text { day ( } 2 \text { weeks) }\end{array}$ & $20.3 \%$ & \\
\hline
\end{tabular}

Abbreviations: R, randomized; DB, double-blind; PC, placebo-controlled; OL, open label; TN, transdermal nicotine.

randomized to receive varenicline $(0.3 \mathrm{mg}$ daily, $1 \mathrm{mg}$ daily, or $1 \mathrm{mg}$ twice daily), bupropion SR $150 \mathrm{mg}$ twice daily or matched placebo. Enrollees in each arm also received brief counseling (approximately 10 minutes) on a weekly basis. Treatment was ongoing for 7 weeks. Study subjects had to have smoked an average of 10 cigarettes daily for the previous year. Abstinence was confirmed by carbon monoxide level evaluation. At 4 weeks, the continuous quit rate (CQR, defined as continuous abstinence from smoking), was $48 \%$ for the varenicline $1 \mathrm{mg}$ twice daily group, and $37.3 \%$ for the $1 \mathrm{mg}$ daily group $(P<0.001 \mathrm{vs}$ placebo for both). In comparison, the bupropion CQR was $33.3 \%$ ( $P<0.002$ vs placebo). The 4-week CQR was thus essentially tripled for the $1 \mathrm{mg}$ twice daily dose of varenicline and doubled for the bupropion SR arm (both vs placebo (17.1\%)). An optional nondrug treatment phase was continued through week 52 , and the results for continued abstinence after week 4 to the end of the study favored varenicline $1 \mathrm{mg}$ twice daily (14.4\%) compared with placebo $(4.9 \%, P=0.002)$. Bupropion users did not maintain a statistically significant $\mathrm{CQR}$ vs placebo at week $52(6.3 \%, P=0.6)$.

Aubin and colleagues conducted a phase III trial of varenicline compared with transdermal nicotine. ${ }^{14}$ The trial was of open label design, and 746 subjects were enrolled. The regular varenicline titration schedule was followed and the drug was given for 12 weeks. The nicotine patch was dosed at $21 \mathrm{mg} /$ day for 6 weeks, and then $14 \mathrm{mg} /$ day and $7 \mathrm{mg} /$ day, each for 2 weeks (total therapy duration for transdermal nicotine was 10 weeks). Subjects using nicotine replacement stopped smoking the day treatment was initiated. Follow up continued to week 52 . The carbon monoxide-confirmed CQR for weeks 9 for 12 significantly favored varenicline (55.9\% vs $43.2 \%$ for nicotine, $P<0.001)$. The CQR at week $52 \mathrm{did}$ not reach statistical significance, but still favored varenicline ( $26.1 \%$ vs $20.3 \%$ vs nicotine, $P=0.056$ ). Potentially, the open label design of the study and the difference in total treatment time ( 2 additional weeks for varenicline) had some effect on the study outcomes.

Two additional phase III trials of identical design were completed to compare varenicline therapy to bupropion SR and placebo. ${ }^{15,16}$ Smokers in both studies were randomized to receive one of the three therapies in addition to brief weekly counseling. All subjects were followed for 52 weeks, 12 of which consisted of drug therapy (or placebo). The number of subjects enrolled in the two studies was nearly identical at $1025^{15}$ and $1027 .{ }^{16}$ The standard varenicline titration schedule was followed. Bupropion SR was administered at a dose of $150 \mathrm{mg}$ daily for the first 3 days, and was then titrated to $150 \mathrm{mg}$ twice daily for the remainder of the active treatment phase. The primary outcome was carbon monoxide-confirmed CQR from weeks 9 to 12 . Subjects in the first study ${ }^{15}$ that were randomized to varenicline achieved abstinence at a rate of $44 \%$ vs $17.7 \%$ for placebo $(P<0.001)$. Results from the second study ${ }^{16}$ were similar (varenicline CQR $43.9 \%$ vs $17.6 \%$ for placebo, $P<0.001$ ). Additionally, CQR was significantly higher vs bupropion SR for both studies $\left(29.5 \%, P<0.001^{15}\right.$ and $\left.29.8 \%, P<0.001 .{ }^{16}\right)$ Of note, the CQR for weeks 9 to 12 was significant for bupropion SR compared to placebo $(P<0.001$, both studies) as well. The first of the secondary endpoints, $\mathrm{CQR}$ at weeks 9 to 24, demonstrated significance for varenicline compared with placebo for both study groups (29.5\% vs $10.5 \%$, $P<0.001^{15}$ and $29.7 \%$ vs $13.2 \%, P<0.001 .{ }^{16}$ ) Varenicline remained significantly more effective than bupropion at this time point as well. The final outcome measure, $C Q R$ at weeks 9-52 again demonstrated superiority for varenicline vs placebo ( $21.9 \%$ vs $8.4 \%, P<0.001^{15}$ and $23 \%$ vs $10.3 \%, P<0.001^{16}$ ). However, the CQR for bupropion in the first study (16.1\%) was not significantly different from varenicline $(P=0.057) .{ }^{15}$ Varenicline maintained superiority in the second study with bupropion users achieving a CQR of $14.6 \%(P=0.004) .{ }^{16}$ 
In addition to the studies above which were designed to evaluate varenicline efficacy, a novel study has been conducted to determine if varenicline could be used to maintain abstinence beyond the standard treatment duration. Varenicline was initially given for the typical 12 weeks of therapy achieving a CQR of $64.1 \%(n=1210) .{ }^{10}$ (This CQR is substantially higher compared to those in other studies with varenicline, likely due to the open label design of the first part of the study). The subjects were subsequently randomized to receive varenicline or placebo for an additional1 2 weeks to determine if continued maintenance therapy resulted in better long-term outcomes. Subjects were followed for 52 weeks. CQR from weeks 13 to 24 was $70.5 \%$ for varenicline compared with $49.6 \%$ for placebo $(P<0.001)$. Varenicline superiority was maintained at 52 weeks with $43.6 \%$ of subjects achieving continued abstinence vs $36.9 \%$ of placebo users $(P=0.02)$. This study demonstrated that prolonged use of varenicline has the potential to result in higher CQR over time. In fact, varenicline is the first medication to demonstrate the ability to prevent relapse prevention in a significant manner.

\section{Cost-effectiveness}

An analysis of the cost-effectiveness of a particular therapy is accomplished by comparing that therapy to alternative interventions with regard to definitive common outcomes such as life-years saved (LYS), quality adjusted life-years (QALY) gained (a measure that takes into account years that are affected by illness and adjusts their value accordingly in comparison to years of good health), or cases of illness or disease prevented. ${ }^{18}$ It is known that smokers who quit decrease their risk of coronary heart disease by $50 \%$ within a year, and that sustaining non-smoking status for 15 years brings the risk equal to that of someone who never smoked. ${ }^{19}$ Similarly, the risk of stroke and lung cancer declines substantially within a decade. The resources saved by decreasing smoking-related morbidity add significantly to the cost-effectiveness of an intervention. In fact, smoking cessation is among the most cost-effective health care interventions, and is considered a criterion standard. ${ }^{20,21}$ There is no universally accepted threshold for cost-effectiveness, but a cost per QALY gained under US\$50,000 is generally considered positive. ${ }^{22}$ Cessation programs consistently achieve results substantially below this dollar amount. There are a number of cost-effectiveness analyses for smoking cessation therapies, but to date only a small number have included varenicline (Table 2).

A cost-benefit analysis of varenicline compared with bupropion was conducted from the perspective of an employer as payer. ${ }^{23}$ Outcomes data from a previously published study were utilized in the analysis. ${ }^{15}$ A decision tree model was utilized to determine the net benefit of therapy over a 12-month time period. The costs were standardized to 2006 dollars. A discount rate, often included in cost-effectiveness analyses that cover an extended time horizon since a given amount of money will be worth more today than in the future, was not done due to the 12-month limitation. Patient counseling was set at US\$20 per visit, and each visit to the prescriber was set at US\$51 using current procedural terminology (CPT) codes. Treatment costs corresponding to each therapy were US\$639.80 (varenicline), US\$598 (generic bupropion SR), US\$717 (brand name bupropion SR), and US\$371 (placebo), each administered for 12 weeks. A 12-month cost savings of US\$5,390 per nonsmoking employee was determined using data previously published, though the estimated cost was modified by the investigators due to the exclusion of morbidity and mortality (again due to the short period of analysis), property damage and maintenance estimates, and involuntary smoking (since most workplaces were now smoke-free). ${ }^{24}$ Varenicline was considered most beneficial in terms of yearly cost savings to the employer (US\$540.60 per nonsmoker), the next most beneficial intervention being generic bupropion SR (US\$269.80 per nonsmoker). A sensitivity analysis (modification of estimated study values to determine if outcomes will be consistent if they are higher or lower than those used in the original analysis) was conducted by the investigators. Twelve-month savings per quitter was varied from US\$500 to US\$6,000. The threshold where varenicline was the most cost-beneficial was US\$1,184. Quit rates $(\mathrm{QR})$ for each treatment group were also varied, and it was determined that the QR for varenicline would have to be less than $17 \%$ for bupropion SR to become more cost-effective (an unlikely scenario given the quit rates reported in the efficacy studies summarized previously). Twelve-week treatment cost variance demonstrated that the cost of varenicline would have to increase to US\$616 per quitter for the drug to lose its advantage over the other therapies.

A second study conducted from the perspective of the Dutch healthcare system estimated the cost per QALY gained and savings in direct costs from a single cessation attempt. ${ }^{25}$ A BENESCO (Benefits of Smoking Cessation on Outcomes) model was used to simulate the consequences of tobacco use and the benefits of quitting (including medication costs and decrease in morbidity and mortality). A hypothetical subject cohort of 884,000 was followed until all had died. Treatment groups included those who used varenicline, bupropion SR, NRT (dosage form not specified), nortriptyline or no medication therapy. Four disease states commonly associated with 


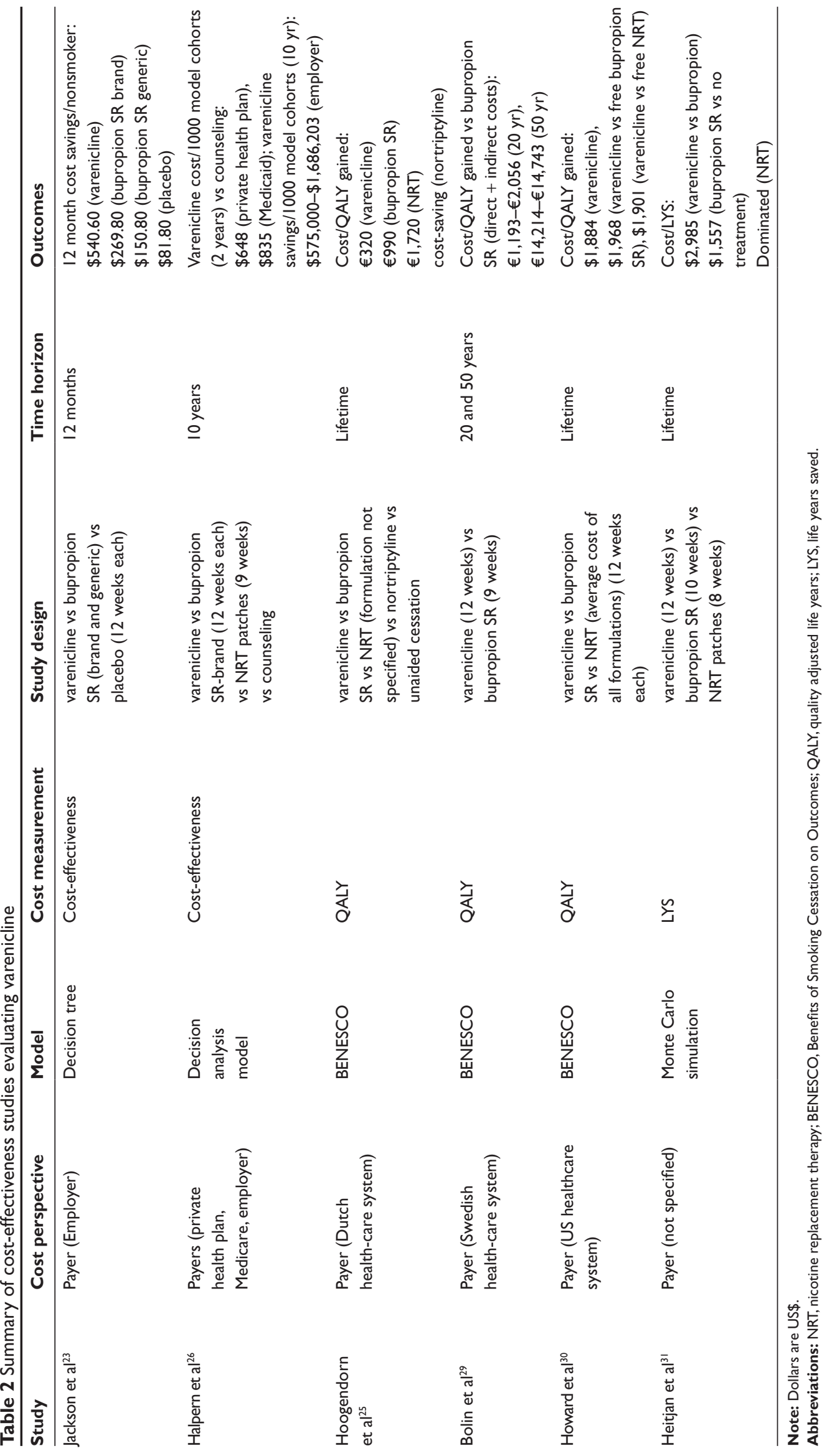


smoking, COPD, lung cancer, coronary heart disease and stroke, were included in estimates of morbidity and mortality. In addition, severe asthma exacerbation was modeled. Though it is certainly possible for a single individual to have more than one of these diseases simultaneously, for the purposes of the study each was mutually exclusive with any subject assumed to have only one at a time. Costs were adjusted to 2004 values, and future costs were discounted at a rate of $4 \%$. In the base model, one in four smokers was expected to make a quit attempt. In terms of number of diseases avoided, varenicline was most favorable. The model allowed for 28,000 incidents avoided in varenicline users, $58 \%$ of those being cases of COPD. Compared with unaided cessation, varenicline cost was $€ 320$ per QALY gained. This was substantially less than bupropion (€990) and NRT (€1,720). In comparison, nortriptyline was cost-saving. Varenicline cost alone (per additional quitter) was higher compared with bupropion and NRT ( $€ 1,350$ and $€ 1,030$ respectively); however, because of superiority in efficacy, the drug remained cost-saving. Compared with nortriptyline, the cost of varenicline per additional quitter was $€ 4,270$, with an additional $€ 1,650$ spent per QALY gained. Despite this higher cost, the price paid per QALY gained with varenicline is still far below the cost-effectiveness threshold previously defined. A sensitivity analysis revealed that increasing the cost per QALY gained to $€ 5,000$ and varying other components of the model would produce results that maintained varenicline as the most cost-effective option more than $80 \%$ of the time vs bupropion SR, NRT, and no drug therapy, and greater than $60 \%$ of the time vs nortriptyline. Of note, the results of the study are believed to be conservative since a limited number of diseases recognized as associated with smoking were included, and because the model did not include exposure of nonusers to tobacco smoke.

Another hypothetical cohort of subjects was followed for 10 years to evaluate the impact of varenicline, brand-name bupropion SR, NRT (patches), or no medication use on private health plans, Medicaid plans, and employer (workplace) cost. $^{26}$ Forty-three percent of subjects were expected to make an attempt at quitting each year. Cessation rates and intervention data were taken from previous studies. The group that was not given drug therapy received intervention in the form of short 10-minute counseling sessions (estimated cost $/$ session $=$ US\$73.04). Both varenicline and bupropion SR were administered for 12 weeks, whereas NRT was given for 9 weeks. Relapse rates were modeled after the 1990 Surgeon General's report on Smoking and Health. ${ }^{27}$ A discount rate of $3 \%$ per year was employed. Based on the model used, an additional $14 \%$ of subjects successfully quit smoking using varenicline compared to bupropion SR. When compared to NRT and no drug therapy, quit rates with varenicline were $25 \%$ and $38 \%$ greater, respectively. Eventual cases of coronary heart disease were estimated to be fewer with varenicline than with other treatments (three, six and nine fewer cases compared to bupropion SR, NRT and no drug therapy respectively). COPD data were similar. Overall, the total cohort cost for varenicline was US\$10,000 less than for bupropion SR. For the other therapies, the cost of varenicline was initially greater (US\$42,000 vs NRT and US\$1 15,000 vs no drug therapy). However, 2 years into the model, the savings in healthcare costs were higher for varenicline compared with both NRT (US\$35,000) and no drug therapy (US\$54,000) owing to the increase in successful quit attempts. For workplace savings (avoidance of absenteeism and lost productivity estimated at 1.59 work days/year per smoker ${ }^{28}$ ), the estimated return on investment (ROI) as decreased costs of healthcare was US\$2.60 for each US\$1 spent on varenicline instead of NRT at 5 years, and US\$6.70 at 10 years. Considering both healthcare costs and workplace costs, the internal rate of return (IRR) was US\$16.90 at 5 years, and US\$34.00 at 10 years for those given varenicline. When all other interventions were considered and both the costs of healthcare and workplace costs were taken into account, the IRR was consistently positive and the ROI was always $>$ US\$1 within the first year for varenicline users. Varenicline proved to be cost saving immediately compared to bupropion SR, and was cost saving within five years for NRT and no drug therapy. Regardless of the payer considered, costs of smoking cessation with varenicline and associated healthcare costs were lower versus bupropion SR. A comparison of varenicline to no drug therapy resulted in the former being cost-effective at 2 years for both the private health plan model (US\$648 per quit) and the Medicaid model (US\$836 per quit). Healthcare and workplace cost savings with varenicline per 1000 model cohorts was US\$575,000 vs bupropion SR, US\$1,106,081 vs NRT, and US\$1,686,203 vs no drug therapy at 10 years.

A study conducted from the perspective of the Swedish economy compared the cost-effectiveness of varenicline and bupropion SR. ${ }^{29}$ A BENESCO model that included indirect costs was used. Monetary values were adjusted to 2003, and a discount rate of 3\% was employed. In the base model, one in four subjects was assumed to attempt to quit smoking, and success rates for varenicline and bupropion $(22.5 \%$ and $15.7 \%$ respectively) were modeled. ${ }^{15,16}$ Varenicline was administered for 12 weeks at a cost (including visits to a general practitioner an motivational support visits) of $€ 452$ 
per subject, and bupropion SR was given for 7 weeks at a cost of $€ 419$. Bupropion users were also provided two additional motivational support visits compared to the varenicline group. Taking both direct and indirect costs into consideration, for each female member of the cohort an additional $€ 1,193$ was spent per QALY gained at 20 years when varenicline was used instead of bupropion. For males, the cost was $€ 2,056$. At 50 years, costs for the varenicline group were $€ 14,214$ and $€ 14,743$ for females and males respectively. Considering only direct costs (ie, costs of healthcare only), varenicline cost per QALY gained was $€ 3,852$ less at 20 years and $€ 3,115$ less at 50 years for females. Varenicline use saved $€ 2,987$ at 20 years and $€ 2,340$ at 50 years for male cohort members. Therefore, when taking only direct costs into account, varenicline was cost saving, and the cost-effectiveness ratio was positive when the net costs of increased survival due to saved morbidity-related costs were considered. There were an estimated 9,200 LYS per 100,000 smokers when varenicline was used instead of bupropion. This number equates to approximately 500 per 100,000 members of the general population. A sensitivity analysis resulted in no change to the overall study conclusions. When varenicline efficacy was decreased to $19 \%$ and cost increased to $€ 2,000$, the maximum cost per QALY gained was achieved (€42,503 for females at 20 years). The investigators noted that there is currently no consensus among decision makers on whether to include or exclude future effects on consumption and production (indirect costs).

Another cost-utility analysis utilizing a BENESCO model was conducted to compare varenicline, bupropion SR, NRT (the cost of which was determined by calculating a weighted average cost for all NRT formulations based on International Medical Statistics prescribing data), and no treatment (not considered to be associated with any cost). ${ }^{30}$ Costs were adjusted to 2005 US dollars, and a discount rate of 3\% per year was applied. The study was conducted from the perspective of the US healthcare system, and only direct costs were taken into account. Per-subject treatment costs were US\$370.96 for varenicline, US\$264.40 for bupropion SR, and US\$405.47 for NRT, each administered for 12 weeks. The assumption used in the base case scenario was that $25 \%$ of the smoking population would attempt a quit in the first year of the model (the only quit attempt considered in the analysis). The cohort $(n=11,925,455)$ was followed for a lifetime horizon. Rates of efficacy were taken from published trials. ${ }^{15,16}$ For the lifetime of the cohort, the incremental cost per QALY gained for varenicline was US\$1,884, a value dominant over all other treatments. When a scenario analysis that assumed zero cost for bupropion and for NRT was conducted, the cost-effectiveness of varenicline remained with an additional US\$1,968 and US\$1,901 spent per QALY gained vs bupropion and NRT respectively. The incremental cost per QALY gained was consistently less than US\$30,000 when 1,000 simulations were run during a probabilistic sensitivity analysis $77.3 \%$ of the time for varenicline versus bupropion, $83.6 \%$ of the time vs NRT, and $82.5 \%$ of the time vs no treatment. Varenicline use was estimated to prevent 143,965 deaths over the lifetime of the model from smoking-related disease (including COPD, coronary heart disease, lung cancer, and stroke), thereby saving additional resources compared to the other treatment options. As in previous models, owing to its superior efficacy as a smoking cessation aid, varenicline resulted in lower expenditures for all direct medical costs (cost of morbidity from disease and cost of cessation therapy). Varenicline costs for the cohort (lifetime) were US\$2,416 million less than bupropion, US\$4,119 million less than NRT and US\$4,741 million less than no treatment.

The final study providing cost-effectiveness data for varenicline was actually conducted to determine the utility of employing pharmacogenetic testing to help choose the best approach to smoking cessation treatment in a given individual. ${ }^{31}$ The model compared varenicline, bupropion SR, NRT (patch), and genetic testing (to choose between bupropion and NRT). Forty-thousand hypothetical smokers were included in the analysis (a Monte Carlo simulation). A sensitivity analysis was completed, and a discount rate of 3\% annually was applied to the base case. Therapy models were NRT for eight weeks, bupropion for ten weeks, and varenicline for 12 weeks, each with minimal counseling. Incremental cost-effectiveness ratios (ICER) were determined to estimate LYS. The ICER of varenicline in the base case scenario was US\$2,985 per LYS (vs bupropion) compared to US\$1,557 per LYS for bupropion (vs no treatment). However, despite being more expensive than bupropion, the investigators found varenicline to be the most cost-effective option due to increased efficacy. The LYS data for genetically tailored therapy and NRT were not given, but they were considered to be dominated by other therapies. The sensitivity analysis demonstrated that the ICERs for varenicline ranged from US\$1,091 to US\$5,381 per LYS compared to the next most effective admissible therapy (bupropion in all but one scenario).

\section{Patient-focused outcomes and safety}

There are many barriers to successful cessation of smoking including concern over weight gain, the fear of relapse, and general lack of support. ${ }^{19}$ However, the greatest barriers are undoubtedly symptoms of withdrawal as the smoker struggles 
to overcome the physical addiction to nicotine and the psychological dependence on the act of smoking itself. One of the potential benefits to varenicline use as demonstrated in the trial by Tonstad et $\mathrm{al}^{17}$ is that the drug appears to possess the potential to help patients maintain abstinence with continued use. However, due to its novel mechanism of action, varenicline users note that even during active treatment the pleasure they receive from using tobacco is diminished, and cravings are tempered.

Subjects in the study by Nides et al had cravings assessed via the Minnesota Nicotine Withdrawal Scale (MNWS) and the Brief Questionnaire of Smoking Urges (QSU-Brief). Investigators found that cravings were significantly diminished in varenicline users compared to placebo users at each week of analysis. ${ }^{13}$ In addition, when asked about the satisfaction they received from smoking, and the enjoyment that smoking provided in terms of respiratory tract sensation, subjects reported a statistically significant decrease (again vs placebo) during the lead in week when they were still using tobacco. Comparable results were reported by Aubin et $\mathrm{al}^{14}$ Subjects in that study reported diminished cravings versus NRT, as well as fewer negative changes in affect and restlessness $(P<0.001$ for all). Another rating scale, the Modified Cigarette Evaluation Questionnaire (mCEQ) produced results demonstrating decreased satisfaction when smoking $(P<0.001)$, decreased psychological reward $(P=0.001)$, less enjoyment of the sensation provided by smoking in the respiratory tract $(P<0.001)$, and significant reduction in craving $(P<0.001)$. However, there was no difference in the aversion subscale. Data from the studies by Gonzalez and Jorenby were similar, MNWS data showing superiority with varenicline vs placebo for urge to smoke, negative affect, and total craving $(P=0.001$ to $P<0.001)$, while mCEQ data were significantly better compared to placebo for smoking satisfaction, psychological reward, enjoyment of respiratory tract sensation and craving reduction $(P=0.04$ to $\mathrm{p}<0.001) .{ }^{15,16}$ QSU-Brief data demonstrated a decrease in total craving score $(P<0.001) .{ }^{15}$

Most adverse effects observed in clinical trials have been mild and transient. A study of 337 subjects was conducted to evaluate the long-term safety of varenicline use. ${ }^{32}$ Subjects were followed for a total of 53 weeks, and varenicline $1 \mathrm{mg}$ twice daily or placebo were given for the first 52 (randomization was completed at a ratio of $2: 1$ ) The overall rate of reported side-effects over the course of the trial for varenicline was $96.4 \%$, and for placebo was $82.5 \%$. Typically, side-effects were noted within the first 4 weeks of the trial, and were mild to moderate. The most common complaint with varenicline treatment was nausea $(40.2 \%)$. Varenicline is known to have mild affinity for serotonin receptors which may contribute to the notable rate at which this side effect occurs. ${ }^{33}$ Nineteen of the subjects that discontinued varenicline prematurely due to side-effects (28.3\% overall in the varenicline group compared with $10.3 \%$ in the placebo group) did so because of nausea. Abnormal dreams were experienced by $22.7 \%$ of varenicline users, and insomnia was a complaint for $19.1 \%$. In clinical trials, insomnia is typically more common in users of bupropion compared to varenicline. ${ }^{13,15,16}$ Of note, both nausea and insomnia are often attributable to nicotine withdrawal. ${ }^{32}$ Elevations in liver enzymes were noted in two varenicline-treated subjects necessitating discontinuation. Hypokalemia led to discontinuation in a third. Only one serious adverse event, a case of bilateral subcapsular cataracts 125 days into the study, was attributed to varenicline by the investigators.

The rate of study withdrawal secondary to adverse events in the trials previously summarized ranged from $1.7 \%$ to $11.2 \%$ with regular varenicline dosing. ${ }^{13-17}$ In comparison, withdrawal rates with placebo were $1.3 \%$ to $9 \%$. Bupropion SR users withdrew at rates of $12.6 \%$ to $15.9 \%$ (consistently higher than varenicline in all studies where the two medications were compared), and nicotine users withdrew at a rate of $4.3 \%$ in the study by Aubin et al. ${ }^{14}$ The most frequent adverse effects were constant among the different studies. As expected, nausea occurred with the highest prevalence ( $28.1 \%$ to $52 \%$ ). Additionally, insomnia (14\% to $35.2 \%$ ), headache $(12.8 \%$ to $24 \%)$, and abnormal dreams $(10.3 \%$ to $15.2 \%$ ) were frequent complaints. ${ }^{13-17}$

In the fourth quarter of 2007, the FDA received nearly 1,000 reports of adverse events that occurred in varenicline users. Seventy-eight patients for whom the drug was prescribed died potentially due to effects related to varenicline. ${ }^{34}$ Concerns about the safety of the drug were raised by the Institute for Safe Medication Practices (a consumer "watchdog" organization). ${ }^{35}$ An early alert was initially put forth in November 2007. Shortly thereafter, a public health advisory was sent out by the FDA warning practitioners about concerns over neuropsychiatric symptoms in patients using varenicline. ${ }^{8}$ Symptoms that had been observed included behavioral changes, mood disorders, generalized agitation, and in some cases, suicidal ideation. At the time, the FDA recommended that prescribers obtain a thorough psychiatric history and advised that monitoring for changes be completed during treatment. ${ }^{36}$ The labeling for varenicline now includes a warning that the drug has been associated with changes in behavior and suicide. (Bupropion labeling also includes a black box warning noting an increase in suicide risk for patients using drugs in the antidepressant class). 
Clinical trial results did not raise concerns about neuropsychiatric side-effects, though most did not allow patients with a history of depression to participate. ${ }^{37}$ One patient in the maintenance therapy trial conducted by Tonstad et al did commit suicide 27 days after completion of the double-blind portion of the study. (The patient had a history of depression that had not been disclosed to the investigators). ${ }^{17}$ Possibly, the size of the studies that were completed prior to varenicline approval were not large enough to detect a trend in neuropsychiatric adverse events. ${ }^{38}$ A causal link between varenicline and suicidal behavior has not been proven, and two out of three patients included in the report from the Institute of Safe Medication Practices were taking other medications that were not ruled out as causes or contributors to symptoms (benzodiazepines most often). ${ }^{34}$ In addition, relative risk must be considered as there were over four million users of varenicline in 2007. It has been hypothesized that some of the psychiatric effects may be related to tobacco withdrawal instead of drug therapy. ${ }^{39}$ However, these adverse events have been noted in patients using varenicline who had not yet ceased smoking, thus ruling out the possibility that withdrawal was solely to blame..$^{36}$ In a study that included both subjects with and without a history of depression, rates of new or worsening symptoms were not increased in the former group. ${ }^{37}$ Depression symptom scores were noted to be similar in both groups at 3 weeks. However, as there were no control groups (with or without a history of depression) that were not exposed to medication, the investigators were not able to discern if an increase in or new onset of depression was attributable primarily to varenicline, nicotine withdrawal, or some other reason. ${ }^{37}$ Since it is not possible to know the underlying etiology of neuropsychiatric disturbances in patients who use varenicline using existing data, it is prudent to engage in increased monitoring in all patients who are actively attempting smoking cessation. ${ }^{39}$

\section{Conclusion}

Various models of analysis have demonstrated that varenicline consistently exceeds existing threshold criteria for costeffectiveness. Though in terms of treatment costs alone varenicline is typically the most costly option among the FDA-approved medications, its superiority in achieving long-term abstinence results in substantial savings because of subsequent avoidance of morbidity and mortality that would otherwise result in increased consumption of healthcare resources. Additionally, varenicline becomes cost-effective within a comparatively short period of time which should allay some of the concerns expressed by payers and employers that they will be unlikely to recoup their investment prior to the time an individual changes plans or employment. Patient acceptance of varenicline is high, and the novel partial agonist has been shown to decrease cravings and withdrawal symptoms, and to blunt the positive reinforcement associated with smoking. The side-effect profile of varenicline is generally mild, transient nausea being the most observed adverse event. Cases of major depression and suicidal ideation have been reported with varenicline use, and although causality has not been definitively proven, caution is warranted if the drug is to be used in an individual with a history of neuropsychiatric disturbance, and patients should be warned about the potential for new onset of symptoms. Overall, given the efficacy, cost-effectiveness, and safety profile of varenicline, it should be considered among the best choices for smoking cessation therapy. However, as with any therapy, individual patient characteristics should be assessed in order to make the most appropriate medication choice.

\section{Disclosures}

The author declares no conflicts of interest.

\section{References}

1. World Bank. Curbing the epidemic: governments and the economics of tobacco control. Washington DC: The World Bank; 1999.

2. Cigarette smoking among adults-United States, 2006. MMWR 2007;56(44):1157-1161.

3. Barendregt JJ, BonneuxL, van der Maas PJ. The health care costs of smoking. N Engl J Med. 1997;337:1052-1057.

4. Doggrell SA. Which is the best primary medication for long-term smoking cessation-nicotine replacement therapy, bupropion or varenicline? Expert Opin Pharmacother. 2007;8:2903-2915.

5. Fiore MC, Jaén CR, Baker JB, et al. Treating tobacco use and dependence: 2008 update. Clinical Practice Guidelines. Rockville, MD: US Department of Health and Human Services; 2008.

6. Potts LA, Garwod GL. Varenicline: the newest agent for smoking cessation. Am J Health Syst Pharm. 2007;64:1381-1384.

7. Harris DS, Anthenaelli RM. Expanding treatment of tobacco dependence. Curr Psychiatry Rep. 2005;7:344-351.

8. Hudmon KS, Corelli RL. ASHP therapeutic position statement on the cessation of tobacco use. Am J Health Syst Pharm. 2009;66:291-307.

9. Warner C, Shoaib M. How does bupropion work as a smoking cessation aid? Addict Biol. 2005;10:219-231.

10. Niaura R, Jones C, Kirkpatrick P. Varenicline. Nat Rev Drug Discov. 2006;5:537-538

11. Tsai ST, Cho HJ, Cheng HS, et al. A randomized, placebo-controlled trial of varenicline, as selective $\alpha_{4} \beta_{2}$ nicotinic acetylcholine receptor partial agonist, as a new therapy for smoking cessation is Asian smokers. Clin Ther. 2007;6:1027-1039.

12. Oncken C, Gonzales D, Nides M, et al. Efficacy and safety of the novel selective nicotinic acetylcholine receptor partial agonist, varenicline, for smoking cessation. Arch Intern Med. 2006;166:1571-1577.

13. Nides M, Oncken C, Gonzales D, et al. Smoking cessation with varenicline, a selective $\alpha_{4} \beta_{2}$ nicotinic receptor partial agonist. Arch Intern Med. 2006;166:1561-1568.

14. Aubin HJ, Britton JR, Oncken C, et al. Varenicline versus transdermal nicotine patch for smoking cessation: results from a randomized openlabel trial. Thorax. 2008;63:717-724. 
15. Gonazales D, Rennard SI, Nides M, et al. Varenicline, an $\alpha_{4} \beta_{2}$ nicotinic acetylcholine receptor partial agonist, vs sustained-release bupropion and placebo for smoking cessation. JAMA. 2006;296:47-55.

16. Jorenby DE, Hays JT, Rigotti NA, et al. Efficacy of varenicline, an $\alpha_{4} \beta_{2}$ nicotinic acetylcholine receptor partial agonist, vs placebo or sustainedrelease bupropion for smoking cessation. JAMA. 2006;296:56-63.

17. Tonstad S, Tonnesen $\mathrm{P}$, Hajek $\mathrm{P}$, et al. Effect of maintenance therapy with varenicline on smoking cessation. JAMA. 2006;296:64-71.

18. Shearer J, Shanahan M. Cost effectiveness analysis of smoking cessation interventions. Aust N Z J Public Heath. 2006;30:428-434.

19. Stack N. Smoking cessation: an overview of treatment options with a focus on varenicline. Pharmacotherapy. 2007;27:1550-1557.

20. Eddy DM. The seven best tests. David Eddy ranks the tests. Harv Health Lett Special Suppl. 1992;17:10-11.

21. Levy DE. Employer-sponsored insurance coverage of smoking cessation treatments. Am J Manag Care. 2006;12:553-562.

22. Eichler HG, Kong SX, Gerth WC, et al. Use of cost-effectiveness analysis in health-care resource allocation decision-making: how are cost-effectiveness thresholds expected to emerge? Value Health. 2004; 7:518-528.

23. Jackson KC, Nahoopii R, Said Q, et al. An employer-based cost-benefit analysis of a novel pharmacotherapy agent for smoking cessation. J Occup Environ Med. 2007;49:453-460.

24. Weis WL. Can you afford to hire smokers? Pers Adm. 1981;26: $71-73,75-78$

25. Hoogendoorn M, Welsing P, Rutten-van Mölken M. Cost-effectiveness of varenicline compared with bupropion, NRT, and nortriptyline for smoking cessation in the Netherlands. Curr Med Res Opin. 2008; 24:51-61

26. Halpern MT, Dirani R, Schmier JK. The cost effectiveness of varenicline for smoking cessation. Manag Care Interface. 2007;20:18-25.

27. Reducing the Health Consequences of Smoking: 25 years of progress. A Report of the Surgeon General. Washington DC, U.S. Department of Health and Human Services publication No. (DHHS)89-8411, 1989.
28. Halpern MT, Shikiar R, Rentz AM, et al. Impact of smoking status on workplace absenteeism and productivity. Tob Control. 2001;10: 233-238.

29. Bolin K, Mörk AC, Willers S, et al. Varenicline as compared to bupropion in smoking-cessation therapy. Cost-utility results for Sweden 2003. Respir Med. 2008;102:699-710.

30. Howard P, Knight C, Boler A, et al. Cost-utility analysis of varenicline versus existing smoking cessation strategies using the BENESCO simulation model. Application to a population of US adult smokers. Pharmacoeconomics. 2008;26:497-511.

31. Heitjan DF, Asch DA, Ray R, et al. Cost-effectiveness of pharmacogenetic testing to tailor smoking-cessation treatment. Pharmacogenomics J. 2008;8:391-399.

32. Williams KE, Reeves $\mathrm{K}$, Billing $\mathrm{CB}$, et al. A double-blind study evaluating the long-term safety of varenicline for smoking cessation. Curr Med Res Opin. 2007;23:793-801.

33. Chantix (varenicline) product information. New York: Pfizer; May 2008.

34. Hays JT. The risk-benefit balance of varenicline for smoking cessation. $J$ Thorac Oncol. 2008;3:949-950.

35. Institute for Safe Medication Practices. Strong safety signal seen for new varenicline risks. Available at: http://www.ismp.org/docs/ vareniclinestudy.asp. Accessed April 14, 2009.

36. Food and Drug Administration. Varenicline information. Available at: http://www.fda.gov/cder/drug/infopage/varenicline/default.htm. Accessed April 11, 2009.

37. McCure JB, Swan GE, Jack L, et al. Mood, side-effects and smoking outcomes among persons with and without probably lifetime depression taking varenicline. J Gen Intern Med. 2009;24:563-569.

38. Kuehn BM. Studies linking smoking-cessation drug with suicide risk spark concerns. JAMA. 2009;301:1007-1008.

39. Spirling LI, Stapleton JA, Sutherland G. Suicidal ideation and varenicline: a possible case of mistaken adverse drug reaction? [letter]. J Clin Psychiatry. 2008;69:1834-1835.
ClinicoEconomics and Outcomes Research

\section{Publish your work in this journal}

ClinicoEconomics \& Outcomes Research is an international, peerreviewed open-access journal focusing on Health Technology Assessment, Pharmacoeconomics and Outcomes Research in the areas of diagnosis, medical devices, and clinical, surgical and pharmacological intervention. The economic impact of health policy and health systems

\section{Dovepress}

organization also constitute important areas of coverage. The manuscript management system is completely online and includes a very quick and fair peer-review system, which is all easy to use. Visit http://www.dovepress.com/testimonials.php to read real quotes from published authors. 